\title{
Der Begriff Wohlbefinden in der Nutztierhaltung - Diskussion aktueller Definitionsansätze als Grundlage für praxisorientierte Forschung am Beispiel Mastschweinehaltung
}

\author{
Herrn Professor Dr. Peter Horst zum 75. Geburtstag gewidmet
}

\begin{abstract}
Title of the paper: Welfare in Farm Animal Husbandry - Current definitions and concepts as basis for practical oriented research with focus on fattening pig husbandry

The paper reviews current definitions and concepts of "welfare" in farm animal welfare related literature, with focus on fattening pig husbandry. The respective concepts of welfare are analysed, and the concepts of explanation are categorised: reductionally, based on the animals' adaptability, based on the animals' feelings, comprehensive. In literature, "welfare" is used non-uniformly. Behind that, there are different concepts of welfare which reflect various attitudes towards animals. Furthermore, varying definitions also lead to differing traits and methods used to estimate welfare in farm animals. This, finally, ends in divergent interpretations of results of such studies even though they seem to pursue similar aims, namely to estimate or promote farm animal welfare. The present analysis shall contribute to the elucidation of backgrounds of interpretation of "welfare" in order to help outline practical oriented investigations dealing with welfare of farm animals, in a clear and well defined manner, in advance. It is emphasized that welfare is complex and any investigation of it, therefore, has to be carried out on a basis of manifold traits (indicators) and methods. Under the premiss of a clear concept and definition of welfare in which the limits are set clearly, too, estimation of welfare of pigs is possible using several indicators.
\end{abstract}

Key Words: welfare, well-being, definition, animal husbandry, pig

\section{Zusammenfassung}

Der Beitrag gibt einen Überblick über aktuell in der Literatur verwendete Definitionsansätze von Wohlbefinden bezogen auf die Nutztierhaltung am Beispiel Mastschweinehaltung. Dabei wird eine Analyse des jeweils zu Grunde gelegten Definitionsansatzes für Wohlbefinden vorgenommen und eine Kategorisierung der Erklärungskonzepte eingeführt: Reduktionistisch, auf Basis der Anpassungsfähigkeit der Tiere, auf Basis der Gefühle der Tiere, umfassend. Der Begriff Wohlbefinden wird in der Literatur uneinheitlich verwendet. Dahinter stecken verschiedene Erklärungskonzepte, die einerseits die unterschiedlichen Einstellungen zu Tieren widerspiegeln und andererseits dazu führen, dass auch Methoden- und Merkmalsauswahl zur Schätzung von Wohlbefinden differieren und entsprechend Ergebnisse trotz vermeintlich gleicher Zielrichtung, Wohlbefinden von Nutztieren zu erfassen oder zu fördern, divergent interpretiert werden. Dabei wird die zu Grunde gelegte Definition nicht immer benannt. Die vorliegende Analyse soll einen Beitrag dazu leisten, Interpretationshintergründe von Wohlbefinden offen zu legen, um praxisorientierte Untersuchungen zu Wohlbefinden von Nutztieren in ihrer Konzeption klar und in ihrer Auslegung von Wohlbefinden exakt beschrieben anzulegen. Es wird herausgestellt, dass Wohlbefinden komplex ist und folglich seine Erforschung nur mit einer vielfältigen Merkmals- (Indikatoren) und Methodenauswahl geschätzt werden kann. Unter einer klaren Konzeption und Definiton von Wohlbefinden, in der auch die Grenzen deutlich gesetzt sind, ist eine Einschätzung des Wohlbefindens von Schweinen anhand mehrerer Indikatoren möglich.

Schlüsselwörter: Wohlbefinden, Wohlergehen, Definition, Nutztierhaltung, Schwein 
1. Einleitung

Für Wohlbefinden gibt es keine einheitliche Definition. Gleichwohl wird der Begriff häufig verwendet, teilweise ohne die Interpretationshintergründe deutlich einzugrenzen oder zu benennen. In der Literatur besteht ein breites Spektrum an Standpunkten dazu, was Wohlbefinden ist. Die vielfältigen Definitionen und Erklärungskonzepte spiegeln zugleich die dahinter stehenden Einstellungen zu Tieren wider. Analog unterscheiden sich auch die vorgeschlagenen oder angewandten Merkmale und Methoden zur Erfassung von Wohlbefinden, was wiederum einen Einfluss auf die Ergebnisse und die daraus gezogenen Schlussfolgerungen haben kann.

Ziel dieses Beitrags ist es, einen Überblick über aktuell in der Welfare-Diskussion verwendete Konzepte zu Wohlbefinden zu geben und die Verknüpfung zwischen Definition oder Erklärung von Wohlbefinden und Merkmals- und Methodenauswahl darzustellen. Dies soll einen Beitrag dazu leisten, praxisorientierte Forschungsvorhaben zu Wohlbefinden von Nutztieren in ihrer Konzeption klar anzulegen. Als Beispiel dient die Mastschweinehaltung. Die Analyse ist Teil eines Forschungsprojektes über Wohlbefinden von Mastschweinen unter besonderer Berücksichtigung ethologischer Merkmale in zwei Haltungssystemen, die einen Kompromiss zwischen Praxisrelevanz und der Erfüllung minimaler Anforderungen an das Wohlbefinden der Mastschweine darstellen (WEBER, 2003). Die Arbeit steht in Zusammenhang mit weiteren Untersuchungen artgerechter Mastschweinehaltung und deren Akzeptanz bei Erzeugern und Verbrauchern in Nordrhein-Westfalen (SCHUBERT, 2003; ZALUDIK, 2002) sowie einer Untersuchung zum Einfluss des Absetzalters auf das Verhalten von Ferkeln nach dem Absetzen (HAARANNEN, 2002).

\section{Definitionsansätze}

Bei der Analyse aktueller Literatur zu Wohlbefinden wird eine Kategorisierung in vier Gruppen von Erklärungskonzepten vorgenommen: Reduktionistisch, auf Basis der Anpassungsfähigkeit der Tiere, auf Basis der Gefühle der Tiere, umfassende Konzepte, in denen Empfindungs- und kognitive Fähigkeiten von Tieren als Grundlage für Wohlbefinden integriert werden. Im Folgenden werden die Begriffe Wohlbefinden (welfare) und Wohlergehen (well-being) synonym verwendet, im Gegensatz zu BROOMs (1993) angesprochener stärkeren Betonung der Gefühlsebene im Begriff well-being (Wohlergehen). Dagegen wird zwischen einerseits Wohlbefinden oder Wohlergehen als auf das Tier und andererseits Tiergerechtheit als direkt auf technische Haltungsdetails bezogenen Begriff unterschieden.

\subsection{Reduktionistische Definitionsansätze}

Stark reduktionistische Ansätze finden sich häufig in praxisorientierter Literatur, wie beispielsweise bei RATSCHOW und CIELEJEWSKI (1996), die sich als Voraussetzung für Wohlbefinden auf die Kriterien Raumtemperatur und Platzangebot beschränken. Beschäftigung der Tiere wird als nicht notwendig angesehen mit der Begründung, Mastschweine müssten keine Zeit mit Nahrungs- und Platzsuche wie Wildschweine verbringen, sondern würden überwiegend ruhen. Auch BÖSCH (2001) spricht von Verbesserung des Wohlbefindens, räumt jedoch den Aufzuchtferkeln nur deshalb Bewegung und Beschäftigung zur Förderung der Bewegung ein, damit sie ein stärkeres Fundament entwickeln, um als Jungsauen den Anforderungen auf Spaltenböden besser gerecht zu werden. BÖSCH (2001) sieht ein gewisses Maß an Druckstellen an den 
Beinen durch die Haltung auf Betonspaltenböden als notwendigerweise in Kauf zu nehmen an. NIGGEMEYER (1999) beschreibt ein sogenanntes „Welfare-System“ für Sauen im Wartebereich, wobei schwerpunktmäßig auf die Sattfütterung der Tiere mit einem quellfähigen, energiearmen Futter aus Automaten eingegangen wird. Eine „u.U. höhere Tiergerechtheit“ wird aufgrund von mehr Ruhe im Stall, weniger Aggressionen und im Gegensatz zur Anbindehaltung mehr Bewegung angenommen.

Diese Sichtweisen sind auf Leistung der Tiere und Rentabilität der Produktion konzentriert. „Wohlbefinden“ wird nicht als eigenständiger Wert berücksichtigt.

In der wissenschaftlichen Literatur gibt es einige Positionen auf der Grundlage, dass erst Krankheit oder präpathologische Zustände Aufschluss über das Wohlbefinden eines Tieres geben können. Einen stark eingegrenzten Standpunkt vertritt MCGLONE (1993), der „sich gut fühlen“ von Tieren nicht als Kriterium für Wohlbefinden akzeptiert. Er erkennt nur Gesundheit als Merkmal für Wohlbefinden an und definiert sie als Krankheitsfreiheit (physisch und mental) und Zustand normaler Physiologie. Ein Tier befinde sich nur im Zustand geringen Wohlbefindens, wenn physiologische Systeme derart gestört sind, dass das Überleben oder die Reproduktion beeinträchtigt sind. Auch bezüglich Schmerz argumentiert MCGLONE (1993), dass er nur das Wohlbefinden beeinträchtige, wenn er ebenfalls die Gesundheit reduziere bzw. das Immunsystem schwäche. In eine ähnliche Richtung, in abgeschwächter Form, geht MOBERG (1993, 1996). Er hält die „Risikoabschätzung“ für ein geeignetes Modell. Für das Wohlbefinden eines Tieres bestehe dann ein Risiko, wenn das Tier Stress erleide, dessen Ausmaß eine bedeutende Abweichung biologischer Ressourcen des Tieres vom Normalzustand verursache. Daher ist für MOBERG (1993, 1996) die Messung präpathologischer Zustände (z.B. Krankheitsanfälligkeit, vermindertes Wachstum, Entwicklung destruktiven Verhaltens) der sinnvollste Parameter zur Bestimmung von Wohlbefinden. PEDERSEN (1996) plädiert für eine Kombination aus präpathologischen Zuständen und physiologischen und ethologischen Stressreaktionen zur Beurteilung von Haltungssystemen hinsichtlich Wohlbefinden. Dabei weist er darauf hin, dass Langzeitstudien zur Untersuchung präpathologischer Zustände in frühen Stadien besonders geeignet seien. Es sei wichtig, längerfristige Stressreaktionen von kurzfristigen Anpassungsreaktionen zu unterscheiden.

Präpathologische Zustände, reduzierte Fitness und Schmerz in Verbindung mit verminderter Gesundheit oder Schwächung der Immunabwehr sind sehr weit reichende Einschränkungen für das Tier und somit nicht geeignet, Stadien, in denen das Wohlbefinden des Tieres in geringerem Ausmaß beeinträchtigt ist, zu erfassen. Darüber hinaus berücksichtigen sie auch nicht die positive Wirkung sogenannten Eustress' auf das Immunsystem, damit die Gesundheit und letztlich das Wohlbefinden von Tieren (TUCHSCHERER und MANTEUFFEL, 2000).

Neuere Konzepte, die auf technische Indikatoren wie Größe, Funktionsfähigkeit und Beschaffenheit von Haltungseinrichtungen, Klimafaktoren sowie Management und Nutzungsform abzielen, sind zunächst unabhängig vom Tier und dienen dazu, die Haltungsumwelt zu beschreiben und mögliche Beeinträchtigungen der Tiere auf Grundlage des Wissens über Körperbau, Körpergröße und Körperfunktion sowie die Erkenntnisse über mögliche Krankheits- und Verletzungsursachen und teilweise auch ethologische Bedürfnisse a priori abzuschätzen (BOCKISCH et al., 1999; DGFZ, 1985; VON BORELL et al., 2001). Für die technischen Indikatoren gilt, dass angegebene Referenzbereiche offen bleiben müssen für neue Erkenntnisse mit dem Ziel, dass 
das Tier in der jeweiligen Haltungsumgebung arttypische Verhaltensweisen ausführen kann (BOCKISCH et al., 1999). Detaillierte Listen technischer Indikatoren auf der Grundlage rechtlicher Vorschriften und Erfahrungen und Empfehlungen aus Praxis und Wissenschaft finden sich bei BOCKISCH et al. (1999), VON BORELL et al. (2001) sowie bei BARTUSSEK (1988) und SUNDRUM et al. (1994), die sogenannte Tiergerechtheitsindices entwickelt haben. Eine Beschreibung von Haltungssystemen anhand technischer Indikatoren und die Anwendung der Kriterienkataloge kann zu einem Vergleich von Haltungssystemen herangezogen werden oder einer unmittelbaren Schwachstellenanalyse auf betrieblicher Ebene dienen (ANDERSSON, 1998; BARTUSSEK, 2000; MATTHES et al., 1998; SUNDRUM, 1995; VON BORELL, 1998). Die Wichtung und Beurteilung (Punktevergabe, Grenzwerte etc.) von Einzelfaktoren integrierender Systeme ist umstritten und wird viel diskutiert (BARTUSSEK, 1999; BRACKE et al., 1999; FEYERLEIN, 1996; HÖRNING, 1998; HÖRNING, 2000b; OFNER et al., 2001). Sowohl KONRAD (1995) als auch SAMBRAUS (1997) und SCIARRA (1998) bemängeln die durch die Errechnung einer Gesamtpunktezahl ungerechtfertigten Kompensationsmöglichkeiten belastender Faktoren durch entlastende, z.B. aus anderen Funktionskreisen. SUNDRUM (1995) hält die Gewichtung zwischen den einzelnen Haltungsparametern für kritisch. Andererseits zeichnen sich Tiergerechtheitsindices aufgrund leicht zu erhebender und quantifizierbarer Indikatoren durch hohe Praktikabilität aus (MATTHES et al., 1998). Ihr Ansatzpunkt ist die Beurteilung technischer und z.T. Management bezogener Ausführungen der Haltungsumwelt. BOCKISCH et al. (1999) machen jedoch darauf aufmerksam, dass der Fokus auf bestimmte haltungstechnische Charakteristika von Haltungssystemen unter Umständen zu falschen Beurteilungen von Aspekten des Wohlbefindens führen kann.

\subsection{Definitionsansätze auf Basis der Anpassungsfähigkeit der Tiere}

Ansätze auf der Basis der Anpassungsfähigkeit des Tieres an seine Umwelt werden von BROOM (1986, 1993, 1996) und WECHSLER (1995) vertreten. Beide sehen in Anpassungsreaktionen des Tieres an seine Umgebung einen normalen Prozess. BESSEI (1984) liefert dazu einen theoretischen Hintergrund und formuliert in Anlehnung an TSCHANZ (1982): „Der Vorgang der Verhaltensanpassung ist die Abstimmung des Verhaltens eines Tieres an seine Umwelt, so dass Selbsterhaltung, Selbstvermehrung und Schadensvermeidung gewährleistet sind“. BESSEI (1984) geht davon aus, dass Säugetiere aufgrund ihrer hoch entwickelten Hirnstruktur sog. „offene“ Verhaltensprogramme besitzen, die es ihnen ermöglichen, Verhaltensabläufe an veränderte Umweltbedingungen so weit anzupassen, dass sie weiterhin den „artgemäßen“ Verhaltensansprüchen entsprechen. Eine Abweichung vom Verhalten der Tiere in ihrer natürlichen Umwelt an sich sei noch kein Anzeichen mangelnden Wohlbefindens, eine Beurteilung erfordere vielmehr gezielte ethologische Experimente. SMIDT et al. (1995) schlagen als Methode für die Einschätzung des Verhaltensbedarfs Wahlversuche und operante Konditionierung vor. Nach der Definition von BROOM (1986) ist Wohlbefinden eines Individuums sein Zustand hinsichtlich seiner Bemühungen, sich mit seiner Umwelt auseinanderzusetzen. „Coping“ bedeutet dabei Kontrolle über mentale und körperliche Stabilität zu haben (FRASER und BROOM, 1997). Da Tiere individuell reagierten und weil jede einzelne Anpassungsreaktion (z.B. Verhaltensänderung) als Indikator zur Erfassung von Wohlbefinden schon allein unzureichendes Wohlbefinden bestätigen könne (BROOM, 1986, 1993), stellt BROOM $(1993,1996)$ 
eine breite Palette an Indikatoren zusammen, anhand derer der Zustand des Tieres auf einem Kontinuum von niedrigem bis zu hohem Wohlbefinden „objektiv“ gemessen werden könne (z.B. Nebennierenrindenaktivitätseffekte, Stereotypien, Wachstum, Krankheitsgeschehen). Gefühle hingen mit den Anpassungsreaktionen zusammen, seien aber noch zu wenig erforscht (BROOM, 1996). Geringes Wohlbefinden sieht BROOM (1993, 1996) dann als gegeben, wenn das Tier Schwierigkeiten hat, sich mit der Umwelt auseinanderzusetzen oder ihm die Anpassung misslingt. Erst letzteren Zustand bezeichnet BROOM (1993) als Stress: „Stress ist ein Umwelteffekt auf ein Tier, der die Kontrollsysteme des Tieres überfordert und seine Fitness reduziert oder dazu tendiert diese zu reduzieren“. Anzeichen für geringes Wohlbefinden sind nach BROOM (1993, 1996), z.B. auf der Basis von Stereotypien, dass über 40 \% des Aktivitätsverhaltens aus Stereotypien bestände. WECHSLER (1995) definiert, dass Wohlbefinden des Tieres solange vorliege, wie es in der Lage sei, aversive Situationen durch effektives Anpassungsverhalten (z.B. Flucht, Suche nach fehlenden Stimuli o.ä.) zu reduzieren. Ein Haltungssystem müsse dem Tier sowohl ermöglichen, die Stress verursachenden Auslöser zu erkennen, als auch erfolgreiche Anpassungsreaktionen auszuführen. Anpassungsverhalten diene dem Tier dazu, proximate, d.h. die unmittelbare Verhaltensorganisation betreffende Ziele zu erreichen und sei somit in der Diskussion um das Wohlbefinden der Tiere ausschlaggebend. Der Erfolg könne anhand der Reduktion physiologischer Stressindikatoren oder von Stereotypien und anderen Verhaltensstörungen gemessen werden. Inwieweit Verhaltensabweichungen einer „Coping“ Strategie oder bereits als anomal einem nicht erfolgreichen „Coping“ (Überforderung der Anpassungsfähigkeit) zugerechnet werden müssten, sei nicht eindeutig geklärt. Durch erfolgreiches „Coping“ werde letztlich die Fitness des Tieres wieder hergestellt (WECHSLER, 1995). Ebenfalls zu den „Coping“ Konzepten gehört VAN PUTTENs (2000) Definiton: „Tierisches Wohlbefinden ist zu verstehen als Leben in angemessener Harmonie mit der Umwelt, sowohl physisch als auch psychisch, d.h. dass die Umwelt qualitativ innerhalb der Anpassungsfähigkeit des Tieres liegen muss“. VAN PUTTENs (1992, 2000) Ansatz ist unmittelbar auf konkrete technische Verbesserungen für die Schweinezucht und das Design von Haltungssystemen ausgerichtet. Somit verbindet VAN PUTTEN (2000) das theoretische Konzept des „Coping“ mit praktischen Hinweisen zur Tiergerechtheit von Haltungssystemen auf der Grundlage der anatomischen Ausstattung von Schweinen sowie ihres vollständigen Ethogramms. Dies bestätigt VAN PUTTENs (1992) Feststellung über tiergerechte Schweinehaltung: Es sei kein Synonym für ideal oder optimal, sondern für eine Situation, mit der ein Tier zurecht kommt.

Die „Coping“ Konzepte setzen ihren Schwerpunkt auf Anpassungen seitens des Tieres. Dass gewisse Anpassungen den Tieren in Tierhaltung abverlangt werden, ist per se folgerichtig (SAMBRAUS, 1997; SMIDT et al., 1995). Es ergibt sich die Frage der Grenzziehung zwischen vertretbaren Anpassungsreaktionen und nicht mehr vertretbaren. Wenn BROOM (1986, 1993) einerseits sagt, dass die moralische Bewertung des Wohlbefindens als eine weitere Größe angesehen werden müsse, die mit der Messung des Wohlbefindens zunächst nichts zu tun habe, andererseits aber von höherem und niedrigerem Wohlbefinden spricht und z.B. für die Stereotypien prozentuale Grenzen am Gesamtaktivitätsverhalten festlegt, dann zeigt sich hier doch ein Widerspruch, denn die Grenzziehung bei 40 \% kann nicht objektiv belegt werden. Sie enthält eine „moralische“ Komponente. Darüber hinaus lässt sich konstatieren, dass bereits die 
Auswahl der Merkmale zur Einschätzung von Wohlbefinden eine a priori Entscheidung darüber beinhaltet, was zu Wohlbefinden gehört. In seiner Übersicht zum Frühabsetzen von Ferkeln kommt auch VON BORELL (2000a) letztlich zu dem Schluss, dass „eine abschließende Bewertung von der Gewichtung und Interpretation der für das Wohlergehen von Sauen und Ferkeln relevanten Indikatoren abhängt“. In der Literatur zur Beurteilung von Haltungssystemen für Schweine bestehen beachtliche Unterschiede der Interpretation, z.B. in der Frage wie viele Abweichungen vom Normalverhalten zulässig sind, die sich nicht zuletzt durch die Auswahl der Merkmale und Methoden ergeben (HÖRNING, 1992, 2000a).

\subsection{Definitionsansätze auf Basis der Gefühle der Tiere}

Einen eindeutigen Schwerpunkt auf die Gefühle des Tieres und dabei speziell das Schmerzempfinden setzt DAWKINS (1988). Sie geht vom Begriff „Leiden“ als die Erfahrung einer großen Spannbreite unangenehmer subjektiver Zustände aus, um tierisches Wohlbefinden zu erfassen. Leiden unterscheide sich von Schmerz dadurch, dass es nicht akut und nicht durch Zeichen des Schmerzes, wie z.B. Schreien, begleitet werde, sondern länger andauere (z.B. Angst, Frustration, Konflikte). Die Messung von Disstress-Schreien (MANTEUFFEL und SCHÖN, 2004) ist somit kein geeignetes Mittel, um Wohlbefinden zu reflektieren. DAWKINS $(1988,1997)$ betont die äußerste Komplexität der mit dem tierischen Wohlbefinden in Zusammenhang stehenden im Tier ablaufenden Prozesse und stellt fest, dass nur eine sehr breite Basis an Merkmalen (z.B. aus den Bereichen Anatomie, Gesundheit, Physiologie, Ethologie und Gehirnforschung) und deren gemeinsame Interpretation dieser Vielschichtigkeit gerecht werden kann. Letztlich könne man jedoch die subjektiven Erfahrungen anderer Spezies nie vollständig entschlüsseln (DAWKINS, 1988, 1997). Wie MCLEAN (2001) hält DAWKINS (1997) die weitere Erforschung mentaler Fähigkeiten der Tiere für wichtig. Andererseits argumentiert DAWKINS (1997) mit BENTHAM (1982), dass es für die Frage der Leidensfähigkeit der Tiere weniger von Bedeutung sei, ob sich die Tiere bewusst sind, als vielmehr, dass sie Leiden empfinden können. Und diese Leidensfähigkeit leitet DAWKINS (1988, 1990) ebenso wie SAMBRAUS (1991) wesentlich aus auf die Wahrnehmung bezogenen Analogien zum Menschen ab. DAWKINS (1990) stellt fest, dass inneres Erleben eines Individuums letztlich nur durch die Annahme gleichen Empfindens vom Menschen wahrgenommen werden könne. SAMBRAUS (1991) äußert umgekehrt: „Der Rückschluss auf Empfindungen wie Schmerz oder Angst bei Tieren aus dem Verhalten ist nicht unsicherer als der auf gleiche Empfindungen anderer Menschen durch verbale Kommunikation“. Auch DUNCAN (1993, 1996) bezieht Wohlbefinden ausschließlich auf Gefühle und somit auf Tiere, die empfindungsfähig sind (höhere Wirbellose und Wirbeltiere). Allerdings beschreibt DUNCAN (1993) ein Gefühl als eine spezielle Aktivität in einem Sinnessystem, die dem Tier bewusst ist (kognitiver Prozess). Wohlbefinden sei weder mit Gesundheit, der Abwesenheit von Stress oder biologischer Fitness gleichzusetzen noch ginge es um Bedürfnisse der Tiere, sondern Wohlbefinden beziehe sich auf die in einer Situation empfundenen negativen und positiven Gefühle, Wünsche oder emotionalen Zustände. Wohlbefinden werde nicht dadurch vermindert, dass ein Tier krank sei, sondern sich krank fühle. Als Methode zur Bestimmung des tierischen Wohlbefindens schlägt DUNCAN (1993) vor, negative Gefühle der Tiere zu erforschen und zu reduzieren und möglicherweise positive zu verstärken. An dieser Stelle räumt er jedoch ein, dass es 
bisher nur indirekte Messmethoden zur „Befragung“ des Tieres nach seinen Gefühlen gebe, z.B. Wahlversuche, und wirft die Frage auf, ob es jemals gelingen wird, durch Messungen im Gehirn von Tieren Gefühle direkter zu erfassen. Aktivitäten limbischer Hirngebiete müssen unter definierten, teilweise extremen Umweltbedingungen erfolgen und können so nur bedingt auf die komplexe Praxis der Tierhaltung übertragen werden (MANTEUFFEL und PUPPE, 1997).

Die auf Gefühlen von Tieren basierenden Ansätze beruhen auf der Annahme von Empfindungsfähigkeit der Tiere und räumen dem Tier als Subjekt einen hohen Stellenwert ein. Allerdings wird auch deutlich, dass Leiden, Schmerz und positive Gefühle nur indirekt erfasst werden können.

$\mathrm{Zu}$ der Frage der Empfindungsfähigkeit von Tieren kommen sowohl LEVEN (1999) als auch SINGER (1996) zu dem Schluss, dass, insbesondere bzgl. Schmerz, keine kategorische Grenze zwischen Mensch und Tier bewiesen werden könne. MARTIN (1996) postuliert, aufgrund der Analogien der Gehirnstrukturen von Mensch und anderen Wirbeltieren, insbesondere des limbischen Systems, sei der Schluss auf homologe Empfindungen, zumindest in Bezug auf die Grundangst, zwingend. SCHEIBE (1997) sieht vor allem in der Untersuchung neurophysiologischer Korrelate einen entscheidenden Fortschritt zur Erfassung homologer emotionaler Zustände. In der Praxis ist die Messung physiologischer Größen möglicherweise selbst aversiv (MANTEUFFEL und PUPPE, 1997), daher muss die Forschung für leicht anwendbare, nicht invasive Methoden verstärkt werden (VON BORELL, 2000b). ROLLIN (1993) urteilt, der „übliche gesunde Menschenverstand“" habe eigentlich nie daran gezweifelt, dass Tiere (ab einer bestimmten Entwicklungsstufe, die landwirtschaftliche Nutztiere in jedem Fall einschließt) einen weiten Bereich an subjektiven Erfahrungen haben; die moralische Bedeutung habe jedoch bis vor kurzem nur eine untergeordnete Rolle gespielt. JENNINGS (1998) kommt zu der Aussage, dass diejenigen, die mit oder über Tiere arbeiten und forschen, wenig Zweifel daran hätten, dass Tiere leidensfähig und bewusst empfindend seien. Selbst wenn anders lautende philosophische Konzepte bestünden, könne man sich einem anthropomorphistischen Vergleich nicht entziehen, und praktische Forschung müsse Bedürfnisse und Präferenzen von Tieren untersuchen (JENNINGS, 1998). TIMBERLAKE (1997) betont in diesem Zusammenhang, die Interpretation tierischen Verhaltens müsse auf der Basis des Wissens über die Tiere beruhen und dürfe nicht menschliche Gefühle und mentale Zustände unmittelbar übertragen. JAMIESON (1993) beobachtet in den letzten Jahrzehnten eine Annäherung zwischen philosophischen Standpunkten. Trotz unterschiedlicher Ausgangstheorien sei eine zunehmende Übereinstimmung dahingehend festzustellen, dass der Mensch seinen Umgang mit nicht-menschlichen Tieren im Sinne der Ermöglichung von mehr Wohlbefinden verbessern müsse (JAMIESON, 1993). Tiere haben einen höheren moralischen Stellenwert in Philosophie, Forschung und Gesellschaft erhalten (JAMIESON, 1993; ROLLIN, 1993). Dabei wird die Förderung positiver Empfindungen von Tieren immer stärker betont (ROLLIN, 1993; FRASER, 1999; TUCHSCHERER und MANTEUFFEL, 2000; VERHOOG, 2000).

\section{4}

Umfassende Definitionsansätze

Auf der Basis der Anerkennung von subjektiven Gefühlen der Tiere wird in einer Reihe von Definitionen von Harmonie zwischen Tier und Umwelt oder sogar trieb- 
haften Intentionen, Erwartungen oder Wünschen der Tiere gesprochen und die Bedeutung positiver Empfindungen für das Wohlbefinden betont.

HURNIK et al. (1985) formulieren: „Wohlbefinden des Tieres ist ein Zustand, in dem physische und psychische Harmonie zwischen dem Organismus und seiner Umgebung besteht; die zuverlässigsten Indikatoren [...] sind Gesundheit und ein normales Verhaltensrepertoire“. HURNIK (1993) schlägt vor, Harmonie auf der Grundlage moralischer Verpflichtung gegenüber empfindungsfähigen und vollständig unter direkter menschlicher Kontrolle stehenden lebenden Organismen wie folgt zu definieren: „Die Möglichkeit für das Lebewesen, sich gemäß seiner genetischen Prädisposition zu einem physisch und psychisch gesunden Organismus zu entwickeln“. Dazu gehört nach HURNIK (1993) die Befriedigung von lebens-, gesundheits- und Komfort erhaltenden Bedürfnissen (Luft, Wasser, Futter, angemessene Unterbringung, z.B. komplex genug, um Langeweile vorzubeugen, effektive Gesundheitsbetreuung, sensibler Umgang usw.). Bedürfnisse sind nach HURNIK et al. (1985) „alle Anforderungen, die ein Organismus für seine normale Entwicklung und Erhaltung physischer und psychologischer Gesundheit benötigt“. Wünsche könnten dagegen auch in einer das Tier schädigenden Weise bestehen. Langlebigkeitsstudien lieferten zuverlässige Informationen für die Güte der Bedürfnisbefriedigung eines Organismus' und damit die Basis für die Verbesserung von Wohlbefinden (HURNIK, 1993). RIST et al. (1989) legen eine weiterreichende Definition für Wohlbefinden zugrunde und fragen, „unter welchen Bedingungen die Tiere sich mit ihrer Umwelt so in Einklang befinden, dass sie ihre (triebhaften) intentionalen Bedürfnisse befriedigen können“. Entscheidend dabei sei „der Grad, in dem sich das Wohlbefinden durch das artgemäße Verhalten offenbart“, d.h. das Tier selbst aktiv werden könne, um Bedürfnisse, z.B. Hunger oder Durst, triebhaft zu befriedigen (RIST et al., 1989). Bei RIST et al. (1989) liegt die Konnotation auf einem wesensgemäßen Umgang mit der Natur. Die arteigenen Bedürfnisse der Tiere sollen nicht primär durch physiologische Parameter ermittelt, sondern durch Beobachten und Durchdenken des Verhaltens der Tiere aufgespürt werden. Bei DANTZER (1993) finden sich insofern ähnliche Aspekte, als er von Erwartungen der Tiere und Bewertungen von Ereignissen durch die Tiere ausgeht. DANTZER (1993) führt aus, dass die Kontrolle und Vorhersagbarkeit von Stressoren für das Wohlbefinden von Tieren wichtiger seien als die physische Art und Beschaffenheit des Stressors. Die Reaktion auf ein Ereignis hänge mehr von den Erwartungen und Bewertungen der Ereignisse durch das Individuum als von den Ereignissen an sich ab. VESTERGAARD (1996) geht dabei so weit, dass er folgert, das alleinige Ausführen einer Verhaltensweise könne wichtiger sein als die funktionale Konsequenz (z.B. Nestbauverhalten der Sau). Neben Art und Dauer eines Stressors beeinflussen Kontrollierbarkeit und Vorhersagbarkeit von Ereignissen physiologische und psychologische Prozesse im Tier (JENSEN et al., 1996; JENSEN und TOATES, 1997). Stressreaktionen der Tiere können deshalb nicht nur anzeigen, wie die Tiere auf ihre Umwelt reagieren, sondern auch einen Einblick in die kognitiven Fähigkeiten der Individuen geben (DANTZER, 1993). DANTZER (1993) hält Stressreaktionen jedoch für noch zu unerforscht und komplex, um damit bereits Gesundheit als Zustand psychologischen und physiologischen Wohlbefindens erklären zu können. Deshalb fordert er weitere Forschung zum Zusammenwirken physiologischer, ethologischer, immunologischer und gehirnfunktioneller Vorgänge im Tier. Auch SANDØE (1996) bezieht Erwartungen und Bewertungen des Tieres in seine Definition ein. Er definiert Wohlbefinden als „erfahrene 
Befriedigung von Präferenzen“. Das Wohlbefinden eines Tieres bestehe in der Übereinstimmung der Präferenz des Tieres in einer Situation (seiner Motivation, seiner Wünsche, seinem Verlangen, seiner Hoffnungen u.a.) und der Wahrnehmung des Tieres von seiner Situation. Das Widersprüchliche an dieser Definition, etwas könne zum Wohlergehen beitragen, ohne dass dies auch als angenehm empfunden werde, diskutiert SANDØE (1996) nicht. Zur Messung hält auch er die Einbeziehung ethologischer und physiologischer Reaktionen für wichtig und betont die Bedeutung positiver Zustände für das Wohlbefinden wie Freude oder Erwartung neben negativen wie Langeweile, Angst und Resignation. SANDØE (1996) stellt die These auf, dass Forschung auf dem Gebiet der Lebensqualität des Menschen eng verwandt mit dem Gebiet tierischen Wohlbefindens sei, womit er indirekt auf Analogien (z.B. bezogen auf Stressreaktionen und Gefühle) hinweist. Er betont aber auch, dass Tiere aufgrund ihrer Sinneswahrnehmung Präferenzen besitzen können, die sich wesentlich von denen des Menschen unterscheiden (akustisch, olfaktorisch). SIMONSEN (1996) führt den Begriff tierische Lebensqualität ein und bringt damit zum Ausdruck, dass die Dauer von Schmerz oder Leiden ein entscheidender Faktor für Wohlbefinden ist. Er sieht positive (Spielen, Appetenzverhalten und nach SAMBRAUS (1978) sog. „triebverzehrende Endhandlung“) und negative (Schmerz, Frustration) Gefühle als Grundlage des Wohlbefindens an. Beide mentalen Erfahrungen trügen zur Sicherung der Homöostase zwischen Tier und Umwelt und damit zu Gesundheit, Überleben und Fitness bei. Die Erfassung von Wohlbefinden basiert SIMONSEN (1996) auf wissenschaftlichem Wissen und praktischer Erfahrung, beispielsweise von Tierhaltern, bezogen auf Verhalten, Gesundheit und Physiologie. HOLTUG (1996) definiert Wohlbefinden als die Befriedigung von Wünschen; je mehr die Wünsche eines Tieres befriedigt seien, desto größer sei sein Wohlbefinden und umgekehrt, je mehr die Wünsche enttäuscht oder versagt würden, desto geringer sei das Wohlbefinden. Im Gegensatz zu SANDØE (1996) spezifiziert HOLTUG (1996) die Wünsche als ultimate Ziele, was beispielsweise den Wunsch nach Gesundheit - im Gegensatz zu dem proximaten Wunsch z.B. ein Gift zu fressen - einschließt. So setzt er sich in der Begrifflichkeit zwar deutlich von HURNIK (1993) ab, stimmt aber wiederum im Ziel der Aussage mit ihm überein, dass zum Wohlbefinden nur beitragen kann, was dem Tier auch längerfristig nicht schade. HOLTUG (1996) führt die Forderung nach Nutzen ein und legt dar, dass Tiere Interessen haben und daher der Respekt ihres Telos (griech.: Ziel, (End) Zweck) Nutzen oder die Vermeidung von Schaden für das Tier beinhalte, so dass das Respektieren ihres Telos' eine berechtigte moralische Bedeutung habe, die direkte Verpflichtungen des Menschen gegenüber den Tieren hervorrufe. Ein Wesen könne in moralischer Hinsicht nur dann Nutzen oder Schaden erfahren, wenn es empfindungsfähig sei. Des Weiteren sei eine Handlung gegenüber einem Tier nur dann von moralischer Bedeutung, wenn sie dem Tier nutze oder schade. Dabei gehe es immer um individuelle Tiere, nicht um Spezies, da - obwohl einer Spezies als Ganzes zwar Nutzen oder Schaden zugefügt werden kann - die Spezies an sich kein bewusstes Wesen sei. Bezogen auf das Wohlbefinden von Tieren argumentiert auch HOLTUG (1996), dass der Mensch nicht nur dazu verpflichtet sei, Leiden von den Tieren abzuwenden, sondern auch positives Wohlbefinden so weit wie möglich zu fördern. 
3. Schlussfolgerungen für Wohlbefinden in praxisorientierter Forschung Die Konzepte und Definitionen zu Wohlbefinden machen deutlich, dass Empfindungsund Leidensfähigkeit, kognitive Prozesse oder Bewusstsein zentrale Punkte in der Frage nach dem Stellenwert des Tieres im Vergleich zum Menschen und den daraus resultierenden moralischen Verpflichtungen des Menschen den Tieren gegenüber sind (ÖZMEN und NIDA-RÜMELIN, 1999).

Mit Blick auf Nutztiere, insbesondere Schweine, kann bewusste Empfindungsfähigkeit unterstellt werden. Die Frage nach weiteren mentalen Prozessen, Erinnerungs- und Unterscheidungsvermögen, Lernfähigkeit und zumindest in Ansätzen vorhandenem Bewusstsein - wenn auch speziell für Schweine vermutet (HEMSWORTH et al., 1996; RUSHEN, 1996; RUSHEN et al., 1999; KENDRICK, 1998; LAUGHLIN et al., 1999; DE JONG et al., 2000) - lässt sich nicht abschließend klären. Sie muss es aber auch nicht, da die Empfindungsfähigkeit der Schweine eine ausreichende Basis für die Notwendigkeit zur Förderung ihres Wohlbefindens darstellt. Um mit HASTEDT (2000) zu sprechen: „Die Frage nach einem angemessenen Verständnis des Tieres wird nicht allein dadurch beantwortet, dass sich der Mensch wissenschaftliche Erkenntnisse über dessen Gehirn verschafft“.

Wohlbefinden umfasst die Abwesenheit negativer sowie das Auftreten positiver Gefühle. Ausgehend davon, dass Tierhaltung unvermeidbar Einschränkungen für die Tiere mit sich bringt, bedeutet die Förderung des Wohlbefindens der Tiere, ein Gleichgewicht zwischen Tier und Haltungsumwelt derart zu gestalten, dass die Tiere nicht längerfristig leiden, positives Empfinden ausleben können (z.B. durch Spielen) und ihre Körperfunktionen (Physiologie inkl. Gesundheit und Leistung, Verhalten) nicht überfordert werden. Komplexität und Subjektivität tierischen Empfindens bedingen, dass Wohlbefinden nicht exakt gemessen, sondern nur durch die Einbeziehung mehrerer Merkmale und Parameter (Indikatoren) geschätzt werden kann. STEIGER (2003) weist darauf hin, dass in ethologischen Untersuchungen nicht ,aufgrund weniger, mit grobem Erfassungsraster erhobener Verhaltensweisen (z.B. nur Liegen, Stehen und Fressen) voreilige Schlussfolgerungen und weit reichende Wertungen über ein Haltungssystem gezogen werden dürfen“. Trotz dieser Schwierigkeiten ist eine Einschätzung des Wohlbefindens z.B. in einem Vergleich praxisnaher Haltungssysteme anhand mehrerer Indikatoren möglich (WEBER, 2003). Voraussetzung ist die klare Eingrenzung und Definition von Wohlbefinden.

Bei einem Vergleich von Haltungssystemen muss die relative Vorzüglichkeit gegen weiter gefasste, weniger restriktive Maßstäbe überprüft werden (DAWKINS, 1988; DAWKINS, 1997; PETERSEN et al., 1995; STOLBA und WOOD-GUSH, 1981). In einem Systemvergleich mit extrem reizarmen Haltungsbedingungen ließen sich ansonsten nur geringfügig besser geeignete Haltungsbedingungen bereits positiv bewerten. Andererseits kann unter Extrembedingungen keine Verbesserung durch minimale Anreicherungen erreicht werden, woraus jedoch nicht generell auf einen mangelnden Effekt der Anreicherung geschlossen werden dürfte (HILL et al., 1998). STEIGER (2003) zählt sogar minimale Strohmengen für Schweine zur Grundausrüstung, erst darüber hinaus gehende Haltungseinrichtungen und -strukturen dürften als Anreicherung bezeichnet werden.

Die für die Schätzung tierischen Wohlbefindens verwendeten Indikatoren müssen methodisch nachvollziehbar erhoben werden. Eine Vermischung z.B. wirtschaftlicher und tierbezogener Indikatoren (DE BAEY-ERNSTEN und GARTUNG, 2000; RAT- 
SCHOW, 2001) kann zu einer verzerrten Bewertung von für das Wohlbefinden von Schweinen günstigeren Haltungssystemen führen. Obwohl Aufwand und Praktikabilität der Erhebung, insbesondere für die Schätzung von Wohlbefinden unter Praxisbedingungen, zu berücksichtigen sind, sollten reduktionistische Definitionsansätze nicht mit der Erforschung und Förderung von Wohlbefinden verwechselt werden. Das Konzept Wohlbefinden sollte nicht für Untersuchungen, die stark beschränkt einen (technischen) Teilaspekt beleuchten, missbraucht werden. Die Beschränkung auf Detailfragen wird dem komplexen Thema Wohlbefinden von Nutztieren nicht gerecht (BUCHHOLTZ, 2003; MANTEUFFEL, 2002). BUCHHOLTZ (2003) warnt vor Reduktionismus, Anthropomorphismus und Konzeptionslosigkeit.

\footnotetext{
Literatur

ANDERSSON, R.:

Der Tiergerechtheitsindex - TGI. Beurteilung der Tiergerechtheit von Haltungssystemen. KTBL 377 (1998), 99-109

BARTUSSEK, $\mathrm{H}$ :

Haltung. In: Haiger, A.; Storhas, R.; Bartussek, H. (Hrsg.): Naturgemäße Viehwirtschaft, 147-243. Ulmer, Stuttgart, 1988

BARTUSSEK, H.:

A review of the animal needs index (ANI) for the assessment of animals' well-being in the housing systems for Austrian proprietary products and legislation. Livestock Prod. Sc. 61 (1999), 179-192

BARTUSSEK, H.:

How to measure animal welfare? The idea of an „Animal Needs Index“ ANI-35L (Tiergerechtheitsindex TGI 35L): A practical tool for assessing farm animal housing conditions on farm level with respect to animals' well being and behavioural needs - Austrian experiences. Proc. 2nd NAHWOA (Network for Animal Health and Welfare in Organic Agriculture) workshop: Diversity of livestock systems and definition of animal welfare, Córdoba, Spanien, 8.-11. Januar 2000, 135-142
}

BENTHAM, J.:

An introduction to the principles of morals and legislation. In: Burns, J.H.; Hart, H.L.A. (Hrsg.): Repr.

BESSEI, W.: ed. London, Athlone Pr. 1970, 1-343. Methuen, London, New York, 1982

Fixierung und Anpassungsfähigkeit des Verhaltens beim Tier. Der prakt. Tierarzt 65 (1984) 3, 226; 228-232

BOCKISCH, F.-J.; JUNGBLUTH, T.; RUDOVSKY, A.:

Technische Indikatoren für die Beurteilung einer tiergerechten Haltung von Rindern, Schweinen und Legehennen. Züchtungskunde 71 (1999) 1, 8-63

BÖSCH, M.: Jungsauen: Druckstellen an den Gelenken sind vermeidbar. SuS 49 (2001) 2, 18-20

BRACKE, M.B.M.; METZ, J.H.M.; SPRUIJT, B.M.:

Overall animal welfare reviewed. Part 2: assessment tables and schemes. Neth. J. Agric. Sc. 47 (1999), 293-305

BROOM, D.M.:

Indicators of poor welfare. Br. vet. J. 142 (1986), 524-526

BROOM, D.M.:

A usable definition of animal welfare. J. Agric. Env. Ethics 6 (1993) Suppl. 1-2, 15-25

BROOM, D.M.:

Animal welfare defined in terms of attempts to cope with the environment. Acta Agric. Scand. Sect. A (Anim. Sc.) Suppl. 27 (1996), 22-28

BUCHHOLTZ, C.:

Die Bedeutung der Ethologie für den Tierschutz - Festrede zur Preisverleihung durch die SchweisfurthStiftung. Akt. Arb. z. artgem. Tierhltg. 2002, KTBL 418 (2003), 73-77

DANTZER, R.:

Research perspectives in farm animal welfare: the concept of stress. J. Agric. Env. Ethics 6 (1993)

Suppl. 1-2, 86-92

DAWKINS, M.S.:

Animal suffering. The science of animal welfare. Chapmann Hall, London, New York, 1988 


\section{DAWKINS, M.S.:}

From an animal's point of view: motivation, fitness, and animal welfare. Beh. and Brain Sc. 13 (1990), 1-61

DAWKINS, M.S.:

D.G.M. Wood-Gush memorial lecture: Why has there not been more progress in animal welfare research? Appl. Anim. Beh. Sc. 53 (1997), 59-73

DE BAEY-ERNSTEN, H.; GARTUNG, J.:

Zukunftsweisende Stallanlagen für die Schweinehaltung. Zukunftsweisende Stallanlagen im Außenbereich, KTBL 397 (2000), 67-98

DE JONG, I.C.; PRELLE, I.T.; VAN DE BURGWAL, J.A.; LAMBOOIJ, E.; MECHIEL KORTE, S.;

BLOKHUIS, H.J.; KOOLHAAS, J.M.:

Effects of environmental enrichment on behavioral responses to novelty, learning, and memory, and the circadian rhythm in cortisol in growing pigs. Physiol. and Beh. 68 (2000), 571-578

DGFZ (DEUTSCHE GESELLSCHAFT FÜR ZÜCHTUNGSKUNDE): Indikatoren für haltungsbedingte Belastungen in der Nutztierhaltung. Züchtungskunde 57 (1985) 3, 153162

DUNCAN, I.J.H.:

Welfare is to do with what animals feel. J. Agric. Env. Ethics 6 (1993) Suppl. 1-2, 8-14

DUNCAN, I.J.H.:

Animal welfare defined in terms of feelings. Acta Agric. Scand. Sect. A (Anim. Sc.) Suppl. 27 (1996), 29-35

FEYERLEIN, I.:

Vergleich zwischen TGI 200 und TGI 35L für Rinder. Vortrag. GÖT (Gesellschaft für Ökologische Tierhaltung) Fachgespräch: Ansätze zur Bewertung tiergerechter Haltungssysteme, Göttingen, 25.10.1996

FRASER, A.F.; BROOM, D.M.:

FRASER, D.:

Farm animal behaviour and welfare. CAB Int., Wallingford, 1997

Animal ethics and animal welfare science: bridging the two cultures. Appl. Anim. Beh. Sc. 65 (1999), 171-189

HAARANNEN, M.:

Einfluss des Absetzalters auf das Verhalten von Ferkeln nach dem Absetzen. Diss. Hohenheim, Cuvillier, Göttingen, 2002

HASTEDT, H.:

Tiere, Menschen und die Ethik der Nutztierhaltung. Arch. Tierz., Dummerstorf 43 (2000) Sonderheft, 218-225

HEMSWORTH, P.H.; VERGE, J.; COLEMAN, G.J.:

Conditioned approach-avoidance responses to humans: the ability of pigs to associate feeding and aversive social experiences in the presence of humans with humans. Appl. Anim. Beh. Sc. 50 (1996), 71-82

HILL, J.D.; MCGLONE, J.J.; FULLWOOD, S.D.; MILLER, M.F.:

Environmental enrichment influences on pig behavior, performance and meat quality. Appl. Anim. Beh. Sc. 57 (1998), 51-68

HOLTUG, N.:

Is welfare all that matters in our moral obligations to animals? Acta Agric. Scand. Sect. A (Anim. Sc.) Suppl. 27 (1996), 16-21

HÖRNING, B.:

Grundsätze artgemäßer Haltung von Schweinen. In: HÖRNING, B.; BAT e.V. (Hrsg.): Alternative Konzepte 78: Artgemäße Schweinehaltung. Müller, Karlsruhe (1992), 41-49

HÖRNING, B.:

Zur Bewertung von Haltungssystemen durch integrierende Konzepte. Proc. DVG (Deutsche Veterinärmedizinische Gesellschaft) Tagung: Tierschutz und Nutztierhaltung, Nürtingen 5.-7.3.1998, 24-37.

HÖRNING, B.:

Alternative Haltungssysteme für Rinder und Schweine. Ber. Lw. 78 (2000a) 2, 193-247

HÖRNING, B.:

Scoring systems to assess housing conditions of farm animals - examples from dairy cows and laying hens. In: Blokhuis, H.J.; Ekkel, E.D.; Wechsler, B. (Hrsg.): Improving health and welfare in animal production. EAAP publ., 102 (2000b), 89-97

HURNIK, J.F.:

Ethics and animal agriculture. J. Agric. Env. Ethics 6 (1993) Suppl. 1-2, 21-35 
HURNIK, J.F.; WEBSTER, A.B.; SIEGEL, P.B.: Dictionary of farm animal behaviour. Univ. Guelph, Guelph, 1985

JAMIESON, D.: Ethics and animals: a brief review. J. Agric. Env. Ethics 6 (1993) Suppl. 1-2, 15-20

JENNINGS, R.C.: A philosophical consideration of awareness. Appl. Anim. Beh. Sc. 57 (1998), 201-211

JENSEN, K.H.; HANSEN, S.W.; PEDERSEN, L.J.: The effect of long-term stress on the hypothalamic-pituitary-adrenocortical axis and the role of the stressor. Acta Agric. Scand. Sect. A (Anim. Sc.) Suppl. 27 (1996), 40-45

JENSEN, P.; TOATES, F.M.: Stress as a state of motivational systems. Appl. Anim. Beh. Sc. 53 (1997), 145-156

KENDRICK, K.M.: Intelligent perception. Appl. Anim. Beh. Sc. 57 (1998), 213-231

KONRAD, S.: Die Rinder-, Schweine- und Legehennenhaltung in Österreich aus ethologischer Sicht. Habil. Wien, 1995

LAUGHLIN, K.; HUCK, M.; MENDL, M.:

Disturbance effects of environmental stimuli on pig spatial memory. Appl. Anim. Beh. Sc. 64 (1999), $169-180$

LEVEN, C.:

Tierrechte aus menschenrechtlicher Sicht. Der moralische Status der Tiere in Vergangenheit und Gegenwart unter besonderer Berücksichtigung des Präferenz-Utilitarismus von Peter Singer. Kovač, Hamburg, Düsseldorf, Diss., 1999

MANTEUFFEL, G.:

Central nervous regulation of the hypothalamic-pituitary-adrenal axis and its impact on fertility, immunity, metabolism and animal welfare - a review. Arch. Tierz., Dummerstorf 45 (2002) 6, 575-595

MANTEUFFEL , G.; PUPPE, B.:

Ist die Beurteilung der subjektiven Befindlichkeiten von Tieren möglich? Eine kritische Analyse aus naturwissenschaftlicher Sicht. Arch. Tierz., Dummerstorf 40 (1997) 2, 109-121

MANTEUFFEL, G.; SCHÖN, P.C.:

STREMODO, ein innovatives Verfahren zur kontinuierlichen Erfassung der Stressbelastung von Schweinen bei Haltung und Transport. Arch. Tierz., Dummerstorf 47 (2004) 2, 173-181

MARTIN, G.:

Zur naturwissenschaftlichen Erfassbarkeit von Leiden bei Tieren - eine Einführung -. Tierärztl. Umschau 51 (1996), 131-136

MATTHES, H.-D.; FREITAG, J.; GOESMANN, M.:

Entwicklung und Anwendung eines Tiergerechtheitsindexes für die Freilandhaltung von Rindern. Arch. Tierz., Dummerstorf 41 (1998), 573-582

MCGLONE, J.J.:

What is animal welfare? J. Agric. Env. Ethics 6 (1993) Suppl. 1-2, 26-36

MCLEAN, A.N.:

Cognitive abilities - the result of selective pressures on food acquisition? Appl. Anim. Beh. Sc. 71 (2001), 241-258

MOBERG, G.P.: Using risk assessment to define domestic animal welfare. J. Agric. Env. Ethics 6 (1993) Suppl. 1-2, 2-7

MOBERG, G.P.:

Suffering from stress: an approach for evaluating the welfare of an animal. Acta Agric. Scand. Sect. A (Anim. Sc.) Suppl. 27 (1996), 46-49

NIGGEMEYER, H.:

Neues Welfare-System sorgt für Diskussion. SuS 47 (1999) 2, 30-31

ÖZMEN, E.; NIDA-RÜMELIN, J.:

Die moralische Abwägung menschlicher und tierlicher Interessen. In: Joerden, J.C.; Busch, B. (Hrsg.): Tiere ohne Rechte? 63-69. Springer, Berlin, Heidelberg, 1999

OFNER, E.; AMON, T.; AMON, B.; BOXBERGER, J.:

Beurteilungsqualität des Tiergerechtheitsindex TGI 35 L 1995/96. Proc. 6. Wissenschaftstagung zum Ökologischen Landbau: Von Leit-Bildern zu Leit-Linien, Freising-Weihenstephan, 6.-8.3.2001, 325328

PEDERSEN, B.K.:

Animal welfare: a holistic approach. Acta Agric. Scand. Sect. A (Anim. Sc.) Suppl. 27 (1996), 76-81

PETERSEN, V.; SIMONSEN, H.B.; LAWSON, L.G.:

The effect of environmental stimulation on the development of behaviour in pigs. Appl. Anim. Beh. Sc. 45 (1995), 215-224 
RATSCHOW, J.-P.:

Umweltschutz und Tierschutz - ein Konflikt? Vortrag (paper). UBA (Umweltbundesamt) - KTBL (Kuratorium für Technik und Bauwesen in der Landwirtschaft) Fachveranstaltung zur Vorstellung des BVT-Konzeptes: Umwelt oder Tierschutz. Wonach richtet sich die „Beste Verfügbare Technik (BVT)“ für die Schweine- und Geflügelhaltung? Berlin, 19.6.2001

RATSCHOW, J.-P.; CIELEJEWSKI, H.:

Vollspaltenboden hat viele Vorteile. Lw. Wbl. Westf.-Lippe 35 (1996), 32-35

RIST, M.; MITARB.; FORSCHUNGSRING FÜR BIOLOGISCH-DYNAMISCHE WIRTSCHAFTSWEISE E.V. (Hrsg.):

Artgemäße Nutztierhaltung. Freies Geistesleben, Stuttgart, 1989

ROLLIN, B.E.:

RUSHEN, J.:

Animal welfare, science, and value. J. Agric. Env. Ethics 6 (1993) Suppl. 1-2, 44-50

Using aversion learning techniques to assess the mental state, suffering, and welfare of farm animals. J. Anim. Sc., 74 (1996) 8, 1990-1995

RUSHEN, J.; TAYLOR, A.A.; DE PASSILLÉ, A.M.: Domestic animals' fear of humans and its effects on their welfare. Appl. Anim. Beh. Sc. 65 (1999), 285303

SAMBRAUS, H.H.:

Nutztierethologie. Parey, Berlin, Hamburg, 1978

SAMBRAUS, H.H.:

Tierschutz, Naturwissenschaft und Ethologie. Arch. Tierz., Dummerstorf 35 (1991) 1/2, 181-192

SAMBRAUS, H.H.:

Tierverhalten - Anzeiger für eine artgerechte Tierhaltung. Arch. Tierz., Dummerstorf 40 (1997) Sonderheft, 26-34

SANDØE, P.:

Animal and human welfare - are they the same kind of thing? Acta Agric. Scand. Sect. A (Anim. Sc.) Suppl. 27 (1996), 11-15

SCHEIBE, K.M.:

Tierschutz und Tierverhalten - eine Analyse aus Sicht der Ethologie. Arch. Tierz., Dummerstorf 40 (1997), 381-398

SCHUBERT, K.:

Akzeptanz praxisüblicher Mastschweinehaltungen - Eine empirische Untersuchung in NordrheinWestfalen auf der Erzeuger- und Verbraucherebene. Diss. Bonn, Wehle, Bad Neuenahr, 2003

SCIARRA, C.:

TGI - Tiergerechtheit in Punktzahl fassbar? Ökol. und Landbau, 10526 (1998) 1, $42-43$

SIMONSEN, H.B.:

Assessment of animal welfare by a holistic approach: behaviour, health and measured opinion. Acta

SINGER, P.: Agric. Scand. Sect. A (Anim. Sc.) Suppl. 27 (1996), 91-96

Animal liberation. Die Befreiung der Tiere. Rowohlt, Reinbek bei Hamburg, 1996

SMIDT, D.; SCHLICHTING, M.C.; LADEWIG, J.; STEINHARDT, M.:

Ethologische und verhaltensphysiologischen Forschung für tiergerechte Nutztierhaltung. Arch. Tierz.

STEIGER, A.: Dummerstorf, 38 (1995) 1, 7-19

Ethische Aspekte in der angewandten Ethologie. Akt. Arb. z. artgem. Tierhltg. 2002, KTBL 418 (2003), 51-60

STOLBA, A.; WOOD-GUSH, D.G.M.:

Verhaltensgliederung und Reaktion auf Neureize als ethologische Kriterien zur Beurteilung von Haltungsbedingungen bei Hausschweinen. Akt. Arb. z. artgem. Tierhltg. 1980, KTBL 264 (1981), 110128

SUNDRUM, A.:

Zur Beurteilung der Tiergerechtheit von Haltungsbedingungen. Proc. DVG (Deutsche Veterinärmedizinische Gesellschaft) Tagung: Lösung von Tierschutzproblemen mittels alternativer Tierhaltungssysteme, Stuttgart Hohenheim 9.-10.3.1995, 23-34

SUNDRUM, A.; ANDERSSON, R.; POSTLER, G. (Hrsg.):

Tiergerechtheitsindex 200 (TGI 200, 1994). Ein Leitfaden zur Beurteilung von Haltungssystemen. Köllen, Bonn, 1994

TIMBERLAKE, W.:

An animal-centered, causal-system approach to the understanding and control of behavior. Appl. Anim. Beh. Sc. 53 (1997), 107-129 
TSCHANZ, B.:

Verhalten, Bedarf und Bedarfsdeckung bei Nutztieren. Akt. Arb. z. artgem. Tierhltg. 1981, KTBL 281 (1982), 114-128

TUCHSCHERER, M.; MANTEUFFEL, G.:

Die Wirkung von psychischem Streß auf das Immunsystem. Ein weiterer Grund für tiergerechte Haltung (Übersichtsreferat). Arch. Tierz., Dummerstorf 43 (2000), 547-560

VAN PUTTEN, G.:

Forschungsergebnisse und Erkenntnisse zur tiergerechten Schweinehaltung. Züchtungskunde, 64 (1992) 3/4, 209-216

VAN PUTTEN, G.:

An ethological definition of animal welfare with special emphasis on pig behaviour. Proc. 2nd NAHWOA (Network for Animal Health and Welfare in Organic Agriculture) workshop: Diversity of livestock systems and definition of animal welfare, Córdoba, Spanien, 8.-11.1.2000, 120-134

VERHOOG, H.:

Defining positive welfare and animal integrity. Proc. 2nd NAHWOA (Network for Animal Health and Welfare in Organic Agriculture) workshop: Diversity of livestock systems and definition of animal welfare, Córdoba, Spanien, 8.-11.1.2000, 108-119

VESTERGAARD, K.S.:

Assessing animal welfare: the significance of causal studies of behaviour at the motivational level. Acta Agric. Scand. Sect. A (Anim. Sc.) Suppl. 27 (1996), 61-63

VON BORELL, E.:

Belange des Tierschutzes in der Rinder- und Schweinehaltung. Züchtungskunde 70 (1998), 436-445

VON BORELL, E.:

Tierschützerische Beurteilung des isolierten Frühabsetzens (Segregated Early Weaning, SEW) beim Schwein - Eine Übersicht. Arch. Tierz., Dummerstorf 43 (2000a) 4, 337-345

VON BORELL, E.:

Mechanismen der Bewältigung von Stress. Arch. Tierz., Dummerstorf 43 (2000b) 5, 441-450

VON BORELL, E.; BOCKISCH, F.-J.; BÜSCHER, W.; HOY, S.; KRIETER, J.; MÜLLER, C.; PARVIZI, N.;

RICHTER, T.; RUDOVSKY, A.; SUNDRUM, A.; VAN DEN WEGHE, H.:

Critical control points for on-farm assessment of pig housing. Livestock Prod. Sc. 72 (2001), 177-184

WEBER, R.E.F.:

Wohlbefinden von Mastschweinen in verschiedenen Haltungssystemen unter besonderer Berücksichtigung ethologischer Merkmale. Diss. Hohenheim, Cuvillier, Göttingen, 2003

WECHSLER, B.:

Coping and coping strategies: a behavioural view. Appl. Anim. Beh. Sc. 43 (1995), 123-134

ZALUDIK, K.:

Bewertung praxisüblicher Mastschweinehaltungen in Nordrhein-Westfalen hinsichtlich der Tiergerechtheit. Diss. Hohenheim, 2002

Eingegangen:14.01.2005

Akzeptiert: 12.09 .2005

Anschriften der Verfasserinnen

Dr. RAGNHILD E.F. WEBER

Oberer Lindweg 26

53129 BONN

E-mail: reggae_w@yahoo.com

Prof. Dr. ANNE VALLE ZÁRATE

Universität Hohenheim

Inst. für Tierproduktion in den Tropen und Subtropen (480a)

Garbenstr. 17

70599 STUTTGART

E-mail: valle@uni-hohenheim.de 